\title{
Inglés por radio: Punto de encuentro entre la Docencia y la Acción Social
}

Inglés por radio: The intersection of Teaching and Social Action

\author{
Mag. Daniela Barrantes ${ }^{I}$ \\ Mag. Carolina González $z^{2}$ \\ Bach. Mariana Valverde 3
}

\begin{abstract}
Resumen
El presente artículo expone el trabajo que se ha llevado a cabo en el proyecto de acción social siglas ED-964 Lenguas Modernas a su Alcance. Este proyecto de extensión docente pretende formar a los radioescuchas, mediante un programa radial, no solo en términos léxicogramaticales del idioma inglés, sino también en aspectos culturales de países de habla inglesa y en temas de interés social relacionados con la realidad costarricense. En este artículo se hace referencia a los antecedentes del proyecto, al uso de la radio en la enseñanza de un segundo idioma y a conceptos relevantes del hacer, quehacer y funcionar del proyecto. Además, se describen el programa, los recursos utilizados, las formas de divulgación del proyecto y la valoración de la experiencia, la cual, de acuerdo con lo expuesto por la representante de un grupo de impacto y las miembros del programa, ha sido muy enriquecedora y con resultados exponenciales, demostrando el alcance del proyecto en términos educativos y de crecimiento social y personal.
\end{abstract}

Palabras clave: contenido informacional, inglés de sobrevivencia, unidades y frases léxicogramaticales, estrategia didáctica, audiencia radial.

\footnotetext{
${ }^{1}$ Universidad de Costa Rica. Docente Escuela de Lenguas Modernas. Colaboradora proyecto ED-964. Correo electrónico: daniela.barrantes@ucr.ac.cr

${ }^{2}$ Universidad de Costa Rica. Docente Escuela de Lenguas Modernas. Colaboradora proyecto ED-964. Correo electrónico: ana.gonzalezramirez@ucr.ac.cr

${ }^{3}$ Universidad de Costa Rica. Docente Escuela de Lenguas Modernas. Responsable proyecto ED-964. Correo electrónico: mariana.valverdevargas@ucr.ac.cr

Recepción: 18/8/17 Aceptación: 8/2/18.
} 


\begin{abstract}
This article describes the work done in the social action project ED-964, Lenguas Modernas a su Alcance. This endeavour aims at helping listeners construct not only their English vocabulary and grammar knowledge but also their cultural background of English speaking countries, as well as relevant topics related to the social context of our country. The paper discusses the background of the project, the use of the radio as a means towards teaching foreign languages, and the most salient concepts related to the work, results, and management of the project. Moreover, readers will also find a description of the radio program, the allocated resources to produce it, outreach activities, and the assessment of the experience. The results, insofar the perspectives of the representative of the benefited group and the instructors in charge of the show, have been quite enriching and successful, thus proving the far-reaching effect of the project in its audience's education, personal growth, and cultural awareness.
\end{abstract}

Key words: carrier content, survival English, lexico-grammatical units and phrases, teaching strategy, radio audience.

\title{
Introducción
}

El proyecto Lenguas Modernas a su Alcance surge de la inquietud de la Escuela de Lenguas Modernas de extenderse a la comunidad y coincide con la inauguración de la nueva frecuencia radiofónica en AM 870 UCR en el año 2009. Esta nueva emisora de corte educativo proporcionó la plataforma para la difusión radial de la iniciativa. Mediante los programas de inglés y francés por radio, la Universidad de Costa Rica fomenta la educación continua en idiomas de los radioescuchas con temas de fondo que propician la crítica, el análisis y el desarrollo humano, además de que, todavía a un nivel básico, representa la oportunidad de mejorar no solo la calidad de vida de los radioescuchas sino además la posibilidad de otras expectativas laborales. Sin duda alguna, el programa se suma en la línea de Acción Social de la Universidad. 


\section{Antecedentes}

En Latinoamérica, el interés de las instituciones de educación superior por tener un papel más activo en el desarrollo social del país se hace presente desde inicios del siglo XVIII con la Reforma Universitaria, “donde se cristaliza un modelo de universidad que incorpora, entre otros aspectos, la función de extensión en toda su dimensión y con características propias" (Menéndez s.f.: 12). Este carácter reformista de la universidad como agente de cambio y progreso social acoge los proyectos con una función social, los cuales ponen al servicio de la sociedad su producción y conocimientos para convertirse en potenciadores de desarrollo y mejoramiento de la calidad de vida de los habitantes.

El compromiso de la Universidad de Costa Rica con la profesionalización de los habitantes se iguala a su participación en el crecimiento social del país a partir del III Congreso Universitario de 1972-1973 (Araya 2013: 374). Desde entonces, la Vicerrectoría de Acción Social (VAS) de la Universidad de Costa Rica se ha propuesto, mediante los proyectos de Extensión Docente, llevar a la sociedad el carácter formativo de esta casa de enseñanza. Dichos proyectos ocurren en el marco de pertinencia que tiene la misión social de la universidad, una construcción de conocimiento colaborativa y con actores, organizaciones e instituciones de la comunidad que pueden aportar en este diálogo de conocimientos y experiencias. Como Tünnermann Bernheim (citado en Ávila Huidobro et al. 2014: 33) explica, "extensión universitaria es la interacción entre universidad y los demás componentes del cuerpo social, a través de la cual ésta asume y cumple su compromiso de participación en el proceso social de creación de la cultura". Este ambiente de interacción se convierte en un espacio de diálogo y de intervención social que "aporta al acto educativo un plus que el espacio áulico no posibilita" (Ávila Huidobro et al. 2014: 66). En este sentido, el proyecto de radio logra alcanzar a un sector de la población desconocido para las aulas, un otro que es el radioescucha: esa persona que está del otro lado del micrófono y con quien se establece una relación más cercana al compartir semana a semana las experiencias de los personajes y los conocimientos del programa.

Menéndez (s.f.: 1) también resalta este carácter constructivista de la extensión docente al definir esta como "una de las funciones esenciales, que conjuntamente con la investigación y la docencia, constituyen los pilares básicos sobre los que se construye un 
modelo de universidad democrática y comprometida socialmente, en su permanente búsqueda hacia la excelencia, pertinencia y equidad social”.

El proyecto Lenguas Modernas a su Alcance considera la Extensión Docente como un "extender" de las cualidades de algo, en este caso el carácter formativo y comunitario de la educación superior. El mismo se enmarca dentro de los lineamientos dictados por la VAS de la Universidad de Costa Rica, al ser un proyecto de educación no formal que promueve la formación permanente de la comunidad, propiciando la difusión del conocimiento al mismo tiempo que contribuye al desarrollo del país a través de la promoción social y el desarrollo comunitario (Universidad de Costa Rica, 2016b).

\section{La radio en la enseñanza de un segundo idioma}

La versatilidad de la radio para la educación no es ajena para sus radioescuchas. Garfinkel (1972) hace un recorrido histórico del uso de la radio en aulas y fuera de ellas con este propósito. Este autor menciona un estudio publicado por The Modern Language Journal llevado a cabo por Lumley (1934), quien expone que la pronunciación de los estudiantes puede mejorarse mediante el uso de programas radiales diseñados y lanzados al aire con tal propósito y que de alguna manera complementan las clases presenciales. La radio para la enseñanza de idiomas ha sido utilizada desde el siglo pasado.

Sin embargo, Lumley (1934) no es el único en publicar estudios relacionados con el uso de la radio en la enseñanza de idiomas. En 1955, la radio educativa japonesa NHK investiga los resultados de programas de inglés complementarios transmitidos por medio de la radio (Garfinkel 1972: 158). Esta investigación arroja frutos positivos a pesar de no especificar las cuatro macro-destrezas del idioma: pues se basa únicamente en las destrezas de comunicación oral. Y es precisamente este vacío el que retoman los investigadores Xoomsai y Ratanamungala (1962 citados en Garfinkel 1972: 58) al aplicar la radio para evaluar las habilidades orales y escritas en inglés de estudiantes de secundaria en Tailandia. Es así como la radio ha sido utilizada como un recurso para enseñar contenidos y mejorar microdestrezas del idioma, como la pronunciación o el uso de tiempos verbales. Los programas de radio del proyecto ED-964 Lenguas Modernas a su Alcance siguen esta tradición y si bien no hay una interacción cara a cara con la audiencia, la magia de la radio contribuye a despertar su imaginación y potenciar su aprendizaje. 
О人ıмв Lu Wimblu, Rev. Estud. Esc. de Psicología UCR, 13(1) 2018 (marzo-junio): 29-46 /ISSN: 1659-2107

Además, la influencia positiva de la radio es tan provechosa que cadenas internacionales como $\mathrm{ABC}$ tienen sus propios programas radiales en frecuencias AM destinados a enseñar francés a estudiantes australianos y a enseñar inglés a estudiantes de Tailandia e Indonesia. De igual manera, la cadena BBC cuenta con numerosos programas radiales que poco a poco han ido evolucionando a radiovisión (un medio innovador que mezcla la radio con otras tecnologías de bajo costo como videos cortos y material de apoyo) (Garfinkel 1972: 161). La Universidad de Costa Rica, por medio de este proyecto de acción social, se mantiene a la vanguardia de medios alternativos que fomentan el desarrollo humano de la población costarricense.

\section{Conceptos}

En el marco de este proyecto de Inglés por Radio, existen algunos conceptos cuya definición es clave para la visualización del panorama completo con respecto al hacer, quehacer y funcionar del mismo.

Primero que todo, se define extensión docente como el "ofrecer algo a la sociedad, intentar enriquecerla en su bagaje cultural, brindarle una herramienta, un conocimiento, una idea, una creación..." (Tauber citado en Menéndez s.f.: 29). El proyecto de extensión docente, ED- 964 Lenguas Modernas a su Alcance, sección de Inglés por Radio, pretende ser portador de un elemento de crecimiento para la población radioescucha. Busca, además, de manera general, proporcionar espacios radiofónicos que promuevan el conocimiento de estructuras gramaticales y vocabulario básicos para la comunicación en inglés, paralelamente a la difusión de la lengua inglesa por medio de temas históricos, contemporáneos y relevantes, los cuales fomentan un estilo de vida amigable con la sociedad. Finalmente, se pretende familiarizar a los radioescuchas con aspectos culturales no solo de Estados Unidos, sino de otros países de habla inglesa, sin dejar de contrastar al mismo tiempo las raíces costarricenses. A través de los objetivos del proyecto, se fortalece el compromiso social de la universidad y le permite a la Radio 870 UCR, cumplir su objetivo de "acercar el conocimiento de la Universidad de Costa Rica a la población que por diversas razones no está directamente relacionada con [dicha institución]" (G. Solano, comunicación personal, 17 de agosto del 2017). 
El proyecto utiliza la radio como medio instrumental para cumplir con sus objetivos. El alcance que brinda esta plataforma al llegar a miles de personas a la vez y acompañarlas en sus hogares, la convierte en un medio masivo que lleva consigo información útil y cuidadosamente diseñada sobre aspectos de cultura y lengua inglesas.

Dado que el programa se transmite a través de la radio, el medio radiofónico influye grandemente sobre el mensaje, obligando a ajustar los contenidos a las características y limitaciones correspondientes. De ahí que la reelaboración del concepto de medio radiofónico de Kaplún (1999: 56) no como "un mero vehículo electrónico que transmite mensajes a distancia", sino como un instrumento es crucial para la comprensión del trabajo en el proyecto, así como su alcance y objetivos.

Se entenderá como la audiencia a los radioescuchas a quienes está dirigido el programa y que escuchan su emisión con regularidad (Terrones 2014: 14). Se asume que este público meta es una audiencia activa que tiene un rol dinámico, donde sus miembros no son simplemente receptores pasivos del mensaje, sino que se convierten en creadores y constructores de su propio conocimiento al interactuar con los contenidos transmitidos y ponerlos en práctica (Barker 2004: 1).

Como un instrumento de gran poder y alcance, el mensaje transmitido a través de la radio debe ser cuidadosamente adecuado a sus características y limitaciones. De ahí que la selección y diseño de los contenidos a transmitir en el programa sea un proceso de cuidadosa consideración y planeamiento, cuyo objetivo debe ayudar a la población radioescucha en la construcción de su conocimiento tanto en temas de la realidad costarricense como en contenidos lingüísticos y gramaticales de la lengua inglesa.

Los temas que trata el programa Inglés por Radio, de acuerdo con la teoría de enseñanza del inglés, son aquellos clasificados como contenido informacional o carrier content (término utilizado en el área de enseñanza del inglés con fines específicos), los cuales se definen como el contexto utilizado para enseñar el lenguaje específico deseado, conocido como contenido real (Dudley-Evans y St John 1998: 11). Además, Richards y Rodgers (2001: 204) definen contenido informacional como el tema del que se aprende o se habla a través de la lengua meta; se aclara que no consiste en el estudio específico de la lengua que se usa para comunicarse. El programa sigue esta línea, pues los temas abordados son tomados de la realidad nacional costarricense, de forma que proveen un contexto común para los oyentes. 
Es a través de estos contenidos que, de forma inductiva, se cubren aspectos relacionados con la lengua inglesa, los cuales pueden incluir elementos léxicos y gramaticales. Es decir, las referencias a la nuestra realidad son el vehículo a través del que se habla en el idioma meta, mas no el objetivo educativo del programa.

Los contenidos lingüísticos transmitidos a través del programa giran en torno al inglés de sobrevivencia. Se define este como los conocimientos mínimos de la lengua inglesa que un individuo necesita conocer para desenvolverse a un nivel muy básico en un ambiente de habla inglesa como lengua generalizada (Popescu 2014: 3). Las herramientas provistas en el programa le permitirían a una persona no familiarizada a profundidad con el inglés llevar a cabo con éxito y ayudar a otros en tareas haciendo uso de este idioma en diferentes tipos de situaciones, tales como solicitar y dar direcciones, identificar comida en el supermercado, lidiar con acciones relacionadas con números y moneda local, y acudir a servicios de salud. Como se observa, las acciones que se pueden llevar a cabo con inglés de sobrevivencia son tareas del diario vivir que toda persona debe ser capaz de realizar para lograr desenvolverse en un determinado contexto. El proyecto Inglés por Radio provee al radioescucha algunas herramientas lingüísticas que le permitirían lidiar con actividades comunes a un nivel básico en inglés.

Dentro de estas herramientas que se le facilitan a la audiencia se encuentran elementos como unidades gramaticales. Morley (2004: 26) define una unidad gramatical como una extensión de lenguaje que conlleva algún patrón gramatical pequeño o que maneja patrones gramaticales de mayor extensión. Las unidades gramaticales que el programa provee al radioescucha son en su mayoría unidades ya preestablecidas que pueden ser fácilmente imitadas y utilizadas en los contextos del diario vivir; por ejemplo, frases y palabras simples, pero de amplio contenido.

De la misma manera, el programa provee al radioescucha de frases léxicas como herramienta en su inglés de sobrevivencia. Nattinger y DeCarrico (2014: 1) definen éstas como construcciones ya preestablecidas de lenguaje que, si bien pueden variar en longitud, son un fenómeno léxico con un significado ya establecido y aceptado. Por ejemplo, se incluyen expresiones para solicitar el precio de objetos: How much is it?; saludos y nivel de formalidad: hi, hello, good morning; y también estructuras más complejas como frases 
idiomáticas: an apple a day keeps the doctor away. Estas frases, afirman Nattinger y DeCarrico (2014: 1), ocurren más frecuentemente que aquellas construcciones elaboradas a partir de cero, de ahí la importancia de mostrar y enseñar su uso contextualizado en el programa. El aspecto semántico (significado) y gramatical (estructura) de estas frases está ya establecido y no suele ser objeto de cambio en el corto plazo, lo cual facilita su aprendizaje y utilización en la vida diaria.

Puesto que el programa de radio posee un elemento auditivo inherente al medio, el concepto de unidad léxica toma también relevancia. En una unidad léxica se consideran no solo los aspectos gramaticales y semánticos ya tratados con anterioridad, sino que se incluyen también las características fonológicas (sonidos) de las palabras y otras construcciones ya preestablecidas como palabras compuestas, verbos frasales y frases idiomáticas (Pavičić Takač 2008: 6). El programa de radio expone a su público meta a las frases y unidades léxicas de forma auditiva, de manera que el radioescucha se familiarice con el sonido de las construcciones, no con su ortografía ni uso en la oración. Este elemento auditivo es clave en el desarrollo del programa y en la construcción de conocimiento que el mismo pretende propiciar en el radioescucha.

Un elemento sumamente valioso del proyecto es su rol en la construcción de vocabulario en inglés. El carácter educativo del programa conlleva la utilización de técnicas de enseñanza de vocabulario como una forma de ayudar al radioescucha en la adquisición de léxico en la lengua meta. Es por esto que el concepto de estrategia didáctica también es necesario definirlo. Una estrategia didáctica es aquella acción planificada con el propósito de ayudar al estudiante a lograr la construcción de su aprendizaje. Es decir, "una estrategia didáctica es, en un sentido estricto, un procedimiento organizado, formalizado y orientado a la obtención de una meta claramente establecida" (Universidad Estatal a Distancia 2013: 1 2).

Algunas de las estrategias didácticas que vale la pena definir en aras de comprender los objetivos y actividades del programa incluyen:

a) Inferencia del significado de unidades léxicas de acuerdo con el contexto en el que ocurren: Puesto que el público meta del programa posee un nivel nulo o sumamente básico del inglés, es indispensable proveer al oyente de un contexto transparente donde le sea posible inferir el significado de una palabra o frase con base en su 
conocimiento previo y contexto situacional. Pavičić Takač (2008: 17) hace hincapié en el rol tan importante que posee la contextualización del vocabulario, así como la exposición del oyente a información comprensible y en cantidades tan amplias como sea posible, de forma que él mismo pueda construir reglas sobre morfología, unidades fraseológicas y otros significados posibles de las unidades léxicas (pues es el contexto mismo el que determina esto).

b) Presentación explícita de nuevos vocablos: Consiste en la enseñanza explícita de unidades léxicas preseleccionadas, en momentos específicos de la lección elegidos por el docente y donde los estudiantes ejercen un rol de receptores de información (Pavičić Takač 2008: 19-20).

Otras estrategias de presentación/introducción de vocabulario que se aplican en el programa son:

c) Relacionar un vocablo y su equivalente en la lengua natal del oyente: Esta equivalencia semántica se establece en los diálogos cuando un personaje no solo utiliza la palabra en inglés, sino que además provee una aproximación del término en español, como forma de asegurarse que otro personaje (y los oyentes) comprendan su significado (Pavičić Takač 2008: 20).

d) Definición de un término: En el programa los términos son definidos a través de sinónimos, antónimos, ejemplos, descripciones o equivalencias. El proyecto sigue las indicaciones de Pavičić Takač (2008: 20), quien establece que las definiciones deben ser simples y claras, y ser complementadas con otros elementos de forma que se fortalezca el desarrollo léxico del oyente y se consolide la retención de los vocablos en la memoria a largo plazo.

e) Repetición de un elemento de vocabulario: Cada programa plantea una serie de unidades léxicas y gramaticales específicas. Una vez que el término ha sido presentado al oyente y contextualizado en el programa, se procura repetir el mismo en el transcurso de los diálogos. Por ejemplo, en el programa titulado "Artesanías costarricenses" (2016), la repetición de la frase "I like you" aclara su significado por medio del contexto: Gabe declara su amor por Marissa utilizando la expresión "I like 
you", a la cual Marissa responde con el primer significado de la frase: me caes bien; sin embargo, se repite la frase de nuevo, pero esta vez con un segundo significado: me gustas — frase con una connotación romántica—. Finalmente, Marissa utiliza la misma frase para corresponder a los sentimientos de Gabe. En este pequeño extracto, la diferenciación de significados es relevante y la repetición de la frase lo hace posible. La repetición proporciona al radioescucha numerosas oportunidades de encuentro con el término, ayudándole a contextualizarlo y afianzarlo en su realidad. Además, como estrategia de consolidación, entre más frecuentes los encuentros contextualizados con una unidad léxica, mayores son las oportunidades del oyente de asegurar la comprensión del término, incrementar su bagaje léxico y consolidar más firmemente el vocablo en su memoria de largo plazo (Pavičić Takač 2008: 21, 23).

Existen otras estrategias de consolidación de vocablos, entre las que se encuentran la repetición mecánica, que algunos personajes del programa utilizan. También la elaboración de un término en relación con lo ya conocido, así como un nivel muy básico de elaboración semántica (Pavičić Takač 2008: 22).

Finalmente, el proyecto Inglés por Radio funciona en el marco de acción universitaria planeada que, de acuerdo con el Estatuto Orgánico de la Universidad de Costa Rica, tiene como uno de sus objetivos el "desarrollar una acción universitaria planificada en pro del mejoramiento continuo para contribuir a elevar el desarrollo humano y la calidad de vida de los habitantes del país" (Consejo Universitario, 1974, artículo 4). En este sentido, el programa apoya a la comunidad radioyente a continuar sus procesos de aprendizaje a través de la educación continua, la cual se entiende como una tendencia en la docencia universitaria (Francis, Revuelta y Badilla 2009: 7), en aras de promover y fortalecer el vínculo universidad-sociedad por medio de la capacitación permanente de sus miembros. Además, la VAS, en su sitio web, establece que:

La Educación Permanente y Continua da cuenta de aquellas acciones educativas dirigidas al desarrollo del ser, para el mejoramiento de la calidad de vida, independientemente de la condición laboral.

Son unas de las formas por las cuales la Universidad de Costa Rica pone a disposición de la población nacional su capacidad académica e institucional para brindar a personas 
de todas las edades y todos los niveles educativos, la oportunidad de seguir formándose en diversos conocimientos, habilidades y destrezas, para su desarrollo humano y profesional. (Universidad de Costa Rica, 2016a).

Es decir, el programa trabaja con el objetivo de estimular a su audiencia meta a continuar aprendiendo a través de las herramientas que el proyecto provee y de compartir los conocimientos de quienes lo producen y ponen en práctica.

\section{Programa de radio}

El proyecto ED-964 Lenguas Modernas a su Alcance, incluyó la división de Inglés por Radio en el año 2009. En ese entonces un grupo de tres colaboradoras docentes de la Escuela de Lenguas Modernas creó la historia de Rosaura, una señora muy valiente que decidió ir a vivir a Estados Unidos con su hermana Isabel, escritora de una revista de comidas, y Naomi, la hija de esta.

El choque cultural no se hace esperar; sin embargo, con la ayuda de sus familiares, Rosaura consigue aprender un tipo de inglés básico que le permite comunicarse en su nuevo ambiente. La historia concluye en el episodio 50 con el regreso de Rosaura a Costa Rica. El programa aborda temas de corte social como, por ejemplo, el empoderamiento de las mujeres, la separación de una pareja sin que afecte a los hijos, los roles de género impuestos por una sociedad, la tenencia responsable de animales, entre muchos otros.

En el año 2015, luego de un rediseño del formato, se incorporan dos profesoras colaboradoras para dar vida a nuevos personajes. La dinámica involucra una nueva historia con personajes que ejemplifican toda la diversidad cultural tan latente en nuestro país. Para tal propósito, se realiza un casting dirigido a la población estudiantil, el cual resulta en la selección de cuatro participantes. Con el trabajo arduo del equipo y la incorporación en el 2016 de dos personajes más, el programa ha crecido, aunque mantiene el componente de contenido de fondo que fomenta el pensamiento crítico.

\section{Producción}

La realización del programa consta de varias fases. En los meses de enero y febrero, en la fase de preproducción, se elabora un planeamiento anual que incluye las fechas de 
grabación, así como los contenidos informacionales y léxico-gramaticales a utilizar. Luego se procede a la redacción y revisión de los guiones. Una vez aprobados por la coordinadora de producción de la radioemisora AM 870 UCR, pasan a la fase de producción. El equipo de trabajo se encarga de realizar las locuciones, y junto con el técnico asignado por la radio, los programas se editan y masterizan para que finalmente, en postproducción, se programen para salir al aire y en la página web de Radioemisoras UCR (http://radios.ucr.ac.cr/radio-

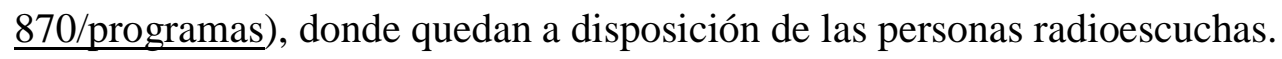

\section{Recursos}

El programa cuenta con la colaboración de la Escuela de Lenguas Modernas y la Radio AM 870 UCR, parte del grupo Radioemisoras UCR. La escuela aporta la carga académica de la coordinadora del proyecto, mientras que las docentes colaboradoras donan voluntariamente su tiempo. De igual modo lo hacen los estudiantes, quienes mediante una invitación y luego de unas pequeñas pruebas, se incorporan al equipo de trabajo de manera voluntaria. La Escuela de Lenguas Modernas también proporciona el material básico como papel e impresión de los guiones. Finalmente, la radio proporciona el espacio físico: el estudio de grabación, la designación de un técnico, la guía por medio de la coordinadora de producción y el apoyo administrativo para la elaboración de una compilación de programas en una serie de discos titulada "Inglés por Radio" y elaborada en convenio con el Sistema Editorial de Difusión Científica de la Investigación (SIEDIN). Sin embargo, los recursos más importantes son la cooperación interdisciplinaria, el idioma y el contexto cultural que se toma en cuenta desde la concepción del programa.

\section{Divulgación}

La divulgación del proyecto se realiza mediante la transmisión de los programas, su disponibilidad en la página web oficial de la radio, las actividades de divulgación por parte de la Comisión de Acción Social de la Escuela de Lenguas Modernas y la distribución por parte de la Asociación de Mujeres Costarricenses.

El primer canal de divulgación del programa es la radio misma a través de la AM 870 UCR, la cual forma parte de las emisoras de Radioemisoras UCR. Es una estación de radio de corte académico cuyo enfoque está en la formación permanente de sus radioescuchas. El 
programa Inglés por Radio se ha transmitido desde el 2009 en horario de jueves a las 11:30 a.m. De la misma forma, en noviembre del 2016 se procedió a subir en la página web oficial de la radio los primeros 30 programas de la serie, de forma que los mismos se pueden escuchar y descargar desde la página de la estación. En el 2017, y a petición de la administración de la radio, se han elaborado cápsulas informativas que rotan en la programación radial y las cuales tienen como finalidad mantener a la audiencia interesada en el programa.

Las actividades de divulgación organizadas por la Comisión de Acción Social (CAS) de la Escuela de Lenguas Modernas en el 2016 también contribuyen a visibilizar el proyecto y el programa. Estas actividades tipo “open house” se realizan en el vestíbulo de la Facultad de Letras y requieren que el proyecto participe con un puesto o stand con información y muestras de los últimos programas. Dichas actividades de la CAS se realizan de una a dos veces por año en la Semana de Bienvenida o entre los meses de octubre y noviembre. Finalmente, la CAS prepara un informe sobre todos los proyectos que presenta en la última Asamblea de Escuela del año. A través de todas estas actividades, la CAS contribuye con la diseminación de la información y cobertura de Lenguas Modernas a su Alcance.

Finalmente, contamos con la participación de la Alianza de Mujeres Costarricenses, quienes distribuyen los discos entre sus miembros. Según su presidenta, Ana Hernández, ésta es una organización no gubernamental que surgió en 1952 en defensa de los derechos de las mujeres y que está conformada por mujeres de distintas partes del país (A. Hernández, comunicación personal, 28 de junio del 2017). En marzo del 2017, 40 discos fueron donados a esta organización con el fin de distribuirlos entre mujeres de diferentes zonas de Costa Rica. De acuerdo con Hernández, los discos se entregan tomando en cuenta las posibilidades de acceso a la tecnología que tengan las compañeras, de forma que solo aquellas miembros que realmente puedan utilizar el insumo obtengan el disco. A su vez, las mujeres que reciben los discos interpretan como parte de su compromiso el buscar la forma de ayudar a las demás compañeras a escucharlos. Es así que se han conformado grupos en distintas provincias para escuchar colectivamente los discos y comentar sus contenidos e información. De esta forma, hasta los familiares de estas mujeres han tenido acceso a los programas (A. Hernández, comunicación personal, 28 de junio del 2017). 


\section{Valoración de la experiencia}

Ser docente en la Universidad de Costa Rica significa nunca dejar de aprender y esta experiencia es sin duda un aprendizaje constante. El ir más allá del aula y enseñar a un grupo diferente al que se acostumbra es sin duda un gran reto que ha valido la pena afrontar. El aprendizaje significativo de las partes involucradas hace que este proyecto de acción social trascienda las barreras físicas y alcance un rango de acción más extenso.

El programa Inglés por Radio conlleva la inmersión del oyente en la cultura costarricense al hacer referencia a temas de la realidad nacional. Para aquellas personas familiarizadas con los eventos de la cotidianidad (o idiosincrasia) costarricense, estos contenidos informacionales les permiten sentirse identificados y motivados a tener un acercamiento con la lengua extranjera, de manera que puedan percibirla como algo accesible a pesar de las limitaciones que los radioescuchas enfrenten para poder aprenderla formalmente. A lo largo de los 50 primeros episodios, el abordaje de diversos temas de la realidad nacional ha sido de gran relevancia para aquellas personas que han tenido la oportunidad de escucharlos, pues les ha permitido no solo aprender a usar el idioma inglés de forma rudimentaria en ciertos contextos de la cotidianidad, sino también tener una visión de mundo más amplia sobre temas de interés social como la no tolerancia a la violencia, tipos de liderazgo, métodos anticonceptivos, matonismo, discriminación, maltrato animal, acoso e importancia de la educación, entre muchos otros. Además, Gioconda Solano, coordinadora de producción de la Radio 870 UCR, opina que "los personajes son cotidianos, las historias agradables, y hay un interesante manejo del humor" (G. Solano, comunicación personal, 17 de agosto del 2017). Estos factores influyen positivamente en la recepción del programa.

Otro elemento muy importante en el diseño de los programas es la relación gramáticavocabulario. La selección de estos componentes léxico-gramaticales debe hacerse de manera cuidadosa, ya que de ello depende en gran medida el éxito del programa. La meticulosa consideración de estos elementos despierta aún más la parte humana y de servicio de las docentes participantes en el proyecto, al tener que estudiar, investigar y acudir a otras realidades para poder calar efectivamente en la realidad de los radioescuchas.

También se toma en cuenta que la audiencia del programa es una audiencia activa que pone en práctica algunos de los contenidos abarcados en el programa y que trata de vivir la utilidad de las frases y vocabulario estudiados al asociarlos con e implementarlos en 
situaciones de su vida y de su realidad. Debemos considerar, entonces, que se trabaja con una audiencia que considera la educación continua como un paso más en su propio desarrollo humano.

El acercamiento de la audiencia al idioma inglés hace esta experiencia muy enriquecedora. Por medio de la radio, los radioescuchas pueden no solo acercarse a la universidad, sino también despertar su interés en seguir adquiriendo aprendizaje mediante nuevos canales. Las estrategias simples pero efectivas eliminan obstáculos sociales como la edad, la educación formal y el tiempo invertido. Este programa ayuda a los radioescuchas a sentirse más familiarizados con el idioma al mismo tiempo que reconocen que el vocabulario es una herramienta muy útil a la hora de expresarse en inglés. La repetición de frases y la contextualización del vocabulario motivan a la población meta a continuar escuchando y aprendiendo por medio de la AM 870 UCR. Podemos encontrar evidencia de la afirmación anterior en la disposición de las mujeres que forman parte de la Alianza de Mujeres, quienes invierten de su tiempo para escuchar los programas en un colectivo que busca el desarrollo humano.

De acuerdo con la información obtenida de la entrevista con la señora Ana Hernández, las integrantes de la asociación, mujeres con iniciativas y metas propias de sus comunidades, no veían como una posibilidad el aprender un segundo idioma por sus condiciones económicas de pobreza. Sin embargo, el programa de radio ha logrado no solo alcanzar a estas mujeres, sino también a sus familias. Les ha abierto la puerta para comunicarse, a un nivel básico, con sus familiares en sus hogares en el idioma meta (A. Hernández, comunicación personal, 28 de junio del 2017). Según Hernández, ha conversado con algunas miembros cuyas madres han intentado comunicarse con ellas en inglés e interpreta esto como señal de que están aprendiendo el idioma a través de los programas (A. Hernández, comunicación personal, 28 de junio del 2017). Este tipo de experiencias muestran cómo el proyecto ha logrado calar en ellas y su iniciativa las ha colocado en una posición proactiva y de crecimiento personal.

De acuerdo con la entrevistada, la importancia de este proyecto "es motivarlas a ellas [las mujeres miembros de la asociación] de las capacidades que ellas tienen, de que pueden y que la sabiduría es innata de todo ser humano y que ellas puedan" (A. Hernández, 
comunicación personal, 28 de junio del 2017). Este conocimiento impacta sus vidas de manera positiva y en palabras de la Sra. Ana Hernández, se transmite a futuras generaciones. El rango de acción entonces se amplía. La solicitud de más discos y la búsqueda de más información con respecto al programa de radio que originó dicho material, evidencia el éxito del programa y su buena recepción entre los oyentes. La señora Hernández nos contó la siguiente anécdota como evidencia del impacto positivo del proyecto. Una miembro de la asociación que asistió a una conferencia en el extranjero le comentó que los discos le ayudaron a sentirse más cómoda en aquellas actividades donde se hablaba inglés, pues se sintió "más ubicada en algunas cosas” (A. Hernández, comunicación personal, 28 de junio del 2017); es decir, se sintió más cómoda con el idioma meta, sus estructuras y sonidos. Está claro que el proyecto consigue una mayor familiarización de la audiencia con el contenido lingüístico del programa, de forma que el inglés es ahora un elemento menos ajeno a su realidad.

Finalmente, el trabajo del proyecto no ha terminado; queda mucho por hacer. Si bien es cierto la evidencia muestra buenos resultados y la experiencia ha sido muy enriquecedora, seguiremos trabajando para mejorar día a día y, mediante la selección de contenidos informacionales pertinentes y de contenidos léxico-gramaticales apropiados, continuar acercando la Universidad a la comunidad costarricense.

\section{Conclusión}

El proyecto, ED-964 Lenguas Modernas a su alcance, en su subdivisión Inglés por Radio, es un programa que nace a partir de la inquietud de la universidad y la Escuela de Lenguas Modernas de trascender y llevar el inglés a las comunidades como una herramienta para mejorar sus posibilidades de movilidad social y sus conocimientos lingüísticos y culturales de países de habla inglesa. Este programa ejemplifica la tendencia mundial de utilizar la radio como medio de difusión y aprendizaje de un idioma. Con un diseño cuidadoso y de la mano con la realidad nacional, se han logrado trabajar exitosamente elementos lingüísticos relacionados con gramática y vocabulario de forma que los radioescuchas se sientan más cómodos y familiarizados con el idioma. Las experiencias de los grupos de impacto demuestran que el proyecto ha logrado llegar a la población a través sus temas actuales, sus estrategias didácticas, y sus historias del diario vivir. Mediante las voces de las 
personas involucradas en el proyecto, el mismo se convirtió en un camino de contacto con la comunidad y de aprendizaje continuo. El éxito del programa motiva a su equipo de trabajo a continuar mejorando, no sólo para cumplir con el compromiso social de la universidad, sino también para ser agentes de cambio entre aquellas personas que escuchan el programa y aprenden con él.

\section{Bibliografía}

Araya, Carlos. (2013). Crecimiento, Democratización y Diversificación de la Educación Superior en Costa Rica (1970-1994). En J. M. Salazar (Ed.), Historia de la Educación Costarricense (pp. 365-407). San José, Costa Rica: EUNED y Editorial de la Universidad de Costa Rica.

Ávila Huidobro, Rodrigo et al. (2014). Universidad territorio y transformación social. Reflexiones en torno a procesos de aprendizaje en movimiento. Avellaneda: UNDAV Ediciones. Recuperado de:

http://ediciones.undav.edu.ar/sites/default/files/Extensi\%C3\%B3n_UNDAV.pdf/

Barker, Chris. (2004). The Sage dictionary of cultural studies. London: Sage Publications.

Consejo Universitario de la Universidad de Costa Rica (1974). Estatuto Orgánico de la Universidad de Costa Rica. Universidad de Costa Rica. Recuperado de: www.cu.ucr.ac.cr/normativ/estatuto_organico.pdf. Consulta: 17 de junio, 2017.

Dudley-Evans, Tony y Maggie St John. (1998). Developments in ESP: A multi-disciplinary approach. Cambridge: Cambridge University Press.

Educación Permanente y Continua. (2016a). Portal web de la acción social de la Universidad de Costa Rica. Recuperado de: https://www.accionsocial.ucr.ac.cr/educacion_continua. Consulta: 19 de julio, 2017. Extensión Docente. (2016b). Portal web de la acción social de la Universidad de Costa Rica. Recuperado de: https://accionsocial.ucr.ac.cr/ed. Consulta: 19 de julio, 2017.

Francis Salazar, Susan, Francisco Revuelta Domínguez Francisco y Eleonora Badilla Saxe, Eleonora. (2009). La docencia universitaria en los espacios virtuales. (1 ed.). San José: Universidad de Costa Rica. 
Garfinkel, Alan. (1972). Teaching Languages via Radio: A Review of Resources. Modern Language Journal, 56(3), 158-162.

Kaplún, Mario. (1999). Producción de programas de radio. El guión-la realización. Quito: Quipus, Ciespal. Recuperado de: http://sitio.amarcuruguay.org/wp-content/uploads/2012/07/LFLACSO-A-KaplunPUBCOM.pdf. Consulta: 13 de julio, 2017.

Menéndez, Gustavo. (s.f.). Desarrollo y conceptualización de la extensión universitaria. Un aporte de la Secretaría de Extensión de la Universidad Nacional del Litoral para al análisis y debate acerca de la extensión universitaria, Universidad Nacional del Litoral. Recuperado de:

$\underline{\text { http://accionsocial.ucr.ac.cr/sites/default/files/documentos/conceptualizacion_menend }}$ ez.pdf. Consulta: 19 de julio, 2017.

Morley, G. David. (2004). Explorations in functional syntax: a new framework for lexicogrammatical analysis. London: Equinox Pub.

Nattinger, James. R. y Jeanette S. DeCarrico. (2014). Lexical phrases and language teaching. Oxford: Oxford University Press.

Pavičić Takač, Višnja. (2008). Vocabulary learning strategies and foreign language acquisition. Clevedon: Multilingual Matters.

Popescu, Daniela. (2014). Teaching survival English to students. Analele Universitatii. Serie Litere Si Stiinte Sociale, (3), 94-99.

Richards, Jack C. y Theodore S. Rodgers. (2001). Approaches and methods in language teaching. Cambridge: Cambridge University Press.

Terrones, Eudoro. (2014). Glosario Radiofónico. Lima. Recuperado de: http://biblioteca.bausate.edu.pe/docs/publicaciones-bausatinas/glosarioradiofonico.pdf. Consulta: 12 de agosto, 2017.

Universidad Estatal a Distancia. (2013). ¿Qué son las estrategias didácticas?. Recuperado de: https://www.uned.ac.cr/academica/images/ceced/docs/Estaticos/contenidos_curso_20 13.pdf. Consulta: 12 de agosto, 2017.

(c) (i) (9)

Esta obra está bajo una licencia de Creative Commons Reconocimiento-NoComercial-CompartirIgual 4.0

\section{Internacional.}

